\title{
A Design Proposal for Gen: Probabilistic Programming with Fast Custom Inference via Code Generation
}

\author{
Marco Cusumano-Towner \\ Probabilistic Computing Project \\ Massachusetts Institute of Technology, USA \\ marcoct@mit.edu
}

\author{
Vikash K. Mansinghka \\ Probabilistic Computing Project \\ Massachusetts Institute of Technology, USA \\ vkm@mit.edu
}

\begin{abstract}
Probabilistic programming languages have the potential to make probabilistic modeling and inference easier to use in practice, but only if inference is sufficiently fast and accurate for real applications. Thus far, this has only been possible for domain-specific languages that focus on a restricted class of models and inference algorithms. This paper proposes a design for a probabilistic programming language called Gen, embedded in Julia, that aims to be sufficiently expressive and performant for general-purpose use. The language provides constructs for automatically generating optimized implementations of custom inference tactics based on static analysis of the target probabilistic model. This paper informally describes a language design for Gen, and shows that Gen is more expressive than Stan, a widely used language for hierarchical Bayesian modeling. A first benchmark shows that a prototype implementation of Gen can be as fast as Stan, only $\sim 1.4 \mathrm{x}$ slower than a hand-coded sampler in $\mathrm{Ju}$ lia, and $\sim 7,500 \mathrm{x}$ faster than Venture, one of the only other probabilistic languages with support for custom inference.
\end{abstract}

CCS Concepts - Mathematics of computing $\rightarrow$ Markovchain Monte Carlo methods; • Software and its engineering $\rightarrow$ Automated static analysis;

Keywords Probabilistic programming, static analysis, code generation, custom inference, inference tactics

\section{ACM Reference Format:}

Marco Cusumano-Towner and Vikash K. Mansinghka. 2018. A Design Proposal for Gen: Probabilistic Programming with Fast Custom Inference via Code Generation. In Proceedings of 2nd ACM SIGPLAN International Workshop on Machine Learning and Programming Languages (MAPL'18). ACM, New York, NY, USA, 6 pages. https://doi.org/10.1145/3211346.3211350

Permission to make digital or hard copies of all or part of this work for personal or classroom use is granted without fee provided that copies are not made or distributed for profit or commercial advantage and that copies bear this notice and the full citation on the first page. Copyrights for components of this work owned by others than the author(s) must be honored. Abstracting with credit is permitted. To copy otherwise, or republish, to post on servers or to redistribute to lists, requires prior specific permission and/or a fee. Request permissions from permissions@acm.org. MAPL'18, June 18, 2018, Philadelphia, PA, USA

( $) 2018$ Copyright held by the owner/author(s). Publication rights licensed to Association for Computing Machinery.

ACM ISBN 978-1-4503-5834-7/18/06 ..\$15.00

https://doi.org/10.1145/3211346.3211350

\section{Introduction}

Probabilistic modeling and inference are widely used in fields such as robotics [26], computer vision [17], statistics [5], cognitive science [11], and artificial intelligence [20]. To formalize and simplify the development of these applications, many probabilistic programming languages have been developed [6-10, 13-16, 18, 21-23, 27, 28, 30]. These languages provide language constructs for probabilistic modeling and either fully or partially automate probabilistic inference given observed data.

A central challenge is providing inference in probabilistic programs that is fast and accurate enough for applications [24]. Thus far, this has only been possible for domain-specific languages that focus on a restricted class of models and inference algorithms. The most prominent example domain is hierarchical Bayesian statistics, in which the BUGS language [25] and the Stan [3] language have each been adopted by thousands of users. Both languages focus on hierarchical Bayesian models over finite sets of random variables, and provide support for a small set of built-in inference algorithms. Another domain where this approach is beginning to succeed is deep generative modeling; prominent examples include Edward [27] and Pyro [14], each of which provides a higher-level interface to models and inference strategies supported by deep learning platforms such as TensorFlow [1] and PyTorch [19].

This paper proposes a design for a probabilistic programming language embedded in Julia that aims to be sufficiently expressive and performant for general-purpose use. Because practitioners typically solve problems using custom inference strategies, the language provides constructs for expressing these strategies.

\subsection{Contributions}

This paper informally introduces a design proposal for a language called Gen, and shows that it is feasible to implement one variant of this design. This language provides constructs for automatically generating optimized implementations of custom inference tactics based on static analysis of the target probabilistic model. This paper uses an example to show that Gen is more expressive than Stan, a widely used language for hierarchical Bayesian modeling. A first benchmark shows that a prototype implementation can be as fast as 
Stan, only $\sim 1.4 \mathrm{x}$ slower than a hand-coded sampler in Julia, and $\sim 7,500 \mathrm{x}$ faster than Venture, one of the only other probabilistic languages with support for custom inference.

\section{Example}

This section illustrates the basic language constructs in Gen for probabilistic modeling and custom inference, using an example Bayesian statistical model for robust regression.

Representing probabilistic models via generative code Figure 1a shows the Gen code for defining a probabilistic model for robust linear regression. The Params struct contains a declaration for each model parameter, including the slope and intercept of the regression function and the noise variance for inliers and outliers. The datum Gen function implements the likelihood model for sampling an output value $\mathbf{y}$ given an input value $\mathbf{x}$ and the params that define the regression function. The regression Gen function implements two additional modeling components: (i) the prior distribution on model parameters, and (ii) the use of datum to generate each y coordinate independently according to the likelihood model.

Several lines of code make stochastic choices that correspond to latent variables in the associated probabilistic model. These lines are annotated with @traced, and terminate in a Julia symbol such as is_outlier. This language construct denotes that the associated expression defines a latent variable whose value should be traced. It also associates the latent variable with a name, relative to the enclosing Gen function. Because each execution of the code generates a randomly sampled realization of all the latent variables in the associated probabilistic model, we will refer to it as "generative code". The overall probabilistic model is defined over the space of all possible executions of the generative code. This basic approach is common to sampling-based probabilistic programming systems $[6,7,14-16,29]$.

Figure $1 \mathrm{~b}$ shows execution trace data structures associated with each of the Gen functions in Figure 1a, and with the invocation of Gen.map. The type signature of these execution traces is inferred by Gen via static analysis of the generative code. Each execution of the Gen functions in Figure 1a produces the data needed to populate one instance of the trace structures from Figure $1 \mathrm{~b}$. The names of the latent variables in the generative code correspond to the field names in the trace data structures.

Generating optimized code for generating traces and mutating them via custom inference tactics Most existing probabilistic programming systems are equipped with one or a handful of "black-box" inference algorithm(s). In contrast, Gen follows Venture [15], a probabilistic programming language that supports custom inference strategies. The use of custom inference strategies is widespread among practitioners, because custom inference strategies can be tailored to reflect the characteristics of the problem at hand. It is this widely understood that custom inference can often deliver much better speed and accuracy than black-box inference.

Gen provides a mechanism for encoding custom inference strategies based on inference tactics that each make stochastic updates to one or more trace data structures. The inference tactics provided by Gen closely follow those provided by Venture and include a broad class of Monte Carlo and gradient search techniques.

Figure 1c shows code that invokes Gen's code generator to create optimized code for initializing and mutating the trace data structures from Figure $1 \mathrm{~b}$ according to specific custom inference tactics, defined in terms of the probabilistic model from Figure $1 \mathrm{a}$.

The simulate operator initializes traces according to the generative code. The observe_y! operator adds an observed output value to the trace corresponding to a specific invocation of datum. The remaining operators apply Markov chain Monte Carlo transition operators to specific latent variables in the model. The latent variable to which an operator applies is specified at code generation time using the reference to the variable within the execution trace (e.g. data[!].y). References can include fields that are unknown at code generation time, like the index (!) into data in data[!].y. This field identifies the specific application of datum relative to which the rest of the reference is resolved. Each '!' placeholder becomes runtime a parameter of the generated operator.

Initializing a Gen trace and adding observed data Figure 1d lines 1-3 show code that loads a training dataset, shown visually in Figure 1e (top left). Line 4 shows code that calls code generated in Figure 1c to generate an initial trace according to the distribution on traces from the generative code in Figure 1a. The trace of this code is visually rendered in Figure 1e (top right). This trace contains a random dataset generated by the generative code. Lines 5-7 show code that adds constraints to trace based on the observed training data; the result is rendered in Figure 1e (bottom left). This trace has the same Params struct as before, and also the same values for the is_outlier variables, but now includes the real values from ys_train. The regression lines, outlier assignments, and y values are thus a poor fit for one another.

Finding a probable trace given the observed data, via a custom inference strategy Once the data has been loaded into a trace, the next step is to use an inference strategy to find probable trace(s) given the observed data. Figure 1d lines 8-16 show code that does this, by invoking a custom inference strategy based on a sequence of inference tactics that stochastically mutate the trace. This custom inference strategy corresponds to a Markov chain Monte Carlo inference algorithm using custom proposals [2]. A single trace produced by one invocation of this code is shown in Figure 1e 


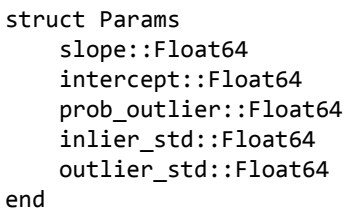

@gen function datum(x: :Float64, params::Params)

mu: :Float64 $=x^{*}$ params.slope + params.intercept

@traced is_outlier::Bool = Gen.flip(params.prob_outlier) : is_outlier std: Float $64=$ is_outlier ? params.inlier_std : params.outlier_std @traced y: :Float64 = Gen.normal(mu, std) :y

end return y

@gen function regression(xs: :Vector\{Float64\})

@traced inlier log var: :Float64 = Gen.normal $(0,1)$ : inlier log var @traced outlier_log_var: :Float64 = Gen.normal $(\theta, 1)$ :outlier_log_var inlier_std::Float64 = sqrt $(\exp ($ inlier_log_var $))$

outlier_std::Float64 = sqrt $(\exp ($ outlier_log_var $))$

@traced slope: :Float64 = Gen.normal $(\theta, \overline{2})$ : slope

@traced intercept: : Float64 = Gen.normal $(0,2)$ : intercept

$\mathrm{n}:$ : Int $=$ length $(\mathrm{xs})$

params: :Params = Params (slope, intercept, 0.5, inlier_std, outlier_std)

@traced ys::Vector $\{$ Float64\} $=\operatorname{Gen} \cdot \operatorname{map}\{$ datum $\}(x s$, fill (params, n)) :data return ys

end

(a) Probabilistic model in the Gen probabilistic modeling language

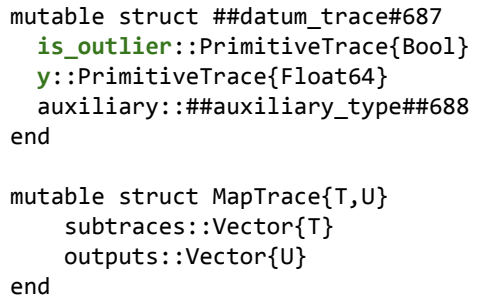

mutable struct \#\#regression_trace\#738 inlier_log_var::PrimitiveTrace\{Float64\} outlier_log_var::PrimitiveTrace\{Float64\} slope: :PrimitiveTrace $\{$ Float64\} intercept::PrimitiveTrace\{Float64\} data: :MapTrace\{\#\#datum_trace\#687,Float64\} auxiliary: :\#\#auxiliary_type\#\#39 end

(b) Static trace types for the model

simulate $=$ load $($ generate_simulate $($ regression $))$

observe_y! = load (generate_observe(regression, : $($ data [! ].y ))

slope_update! = load(generate_metropolis_update(regression, :(slope), (value) -> value + randn() $* 0.5))$

intercept_update! = load(generate_metropolis_update $($ regression, : (intercept), (value) $->$ value + randn ()$* 0.5))$

inlier_log_var_update! = load(generate_metropolis_update(regression, :(inlier_log_var), (value) -> value + randn() * 0.5))

outlier_log_var_update! = load(generate_metropolis_update(regression, :(outlier_log_var), (value) -> value + randn() * 0.5$)$ )

outlier_update! = load(generate_boolean_gibbs_update(regression, :(data[!].is_outlier)))

(c) Generating custom inference tactics specialized to the model using Gen's inference tactic code generators

\# load train data set

1: train_df = CSV.read("train.csv")

2: $x$ s train $=$ train $d f[: x s]$

3: ys_train = train_df $[: y s]$

\# generate an initial trace from the prior 4: $($ trace, _) $=$ simulate (xs_train)

\# observe the training data

5: for $(i, y)$ in enumerate(ys_train)

6: observe_y!(trace, i, y)

7: end

8: for $i=1: 1000$

slope_update! (trace)

intercept_update! (trace)

for $j=1$ : length(xs_train)

end

outlier_update! (trace, j)

inlier_log_var_update! (trace)

outlier_log_var_update! (trace)

16: end

(d) Using generated inference tactics at runtime

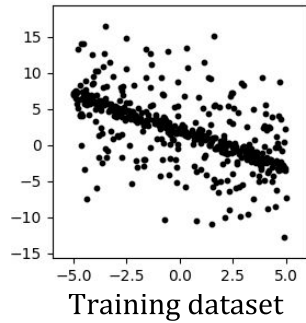

(d, lines 1-3)

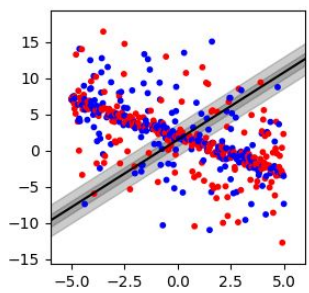

Trace after observing data $(d$, lines 5-7)

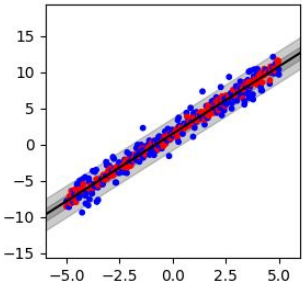

Initial trace sampled from prior $(d$, line 4$)$

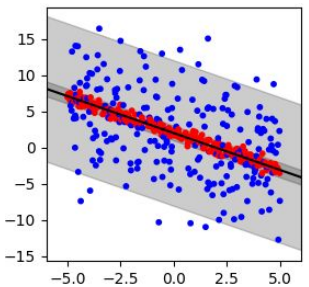

Trace after inference

(d, lines 8-16)

(e) Visualization of the trace at various points in (d). Color indicates the inferred inlier/outlier class. Grey shading shows two standard deviations of inferred noise levels.

Figure 1. Gen applied to a robust Bayesian regression inference problem. 
(bottom right). Now the outlier assignments and model parameters match the observed data.

\section{Benchmarking a Prototype Implementation}

This section compares Gen's expressiveness and performance properties relative to existing probabilistic programming systems on the robust Bayesian linear regression problem illustrated in Figure 1. We compared Gen with Venture [15], Stan [3], Edward [27], and a hand-coded sampler implementation in Julia. A training data set of about 500 data points is shown in Figure 1(e). The data contains a set of low-noise 'inliers' and a set of high-noise 'outliers'. We use the model defined by the Gen program in Figure 1(a). We measure accuracy using predictive likelihood on a held-out test set of about 500 points. Achieving high predictive likelihood requires identifying the inliers and outliers, the latent line, and the amount of inlier and outlier noise, as visualized in Figure 1(e).

We implemented a compositional inference algorithm ('MH+Gibbs') in Gen for this model that uses random-walk Metropolis updates on the real-valued random variables and Gibbs sampling updates on the Boolean is_outlier variables, shown in Figure 1(d). We implemented the same algorithm in Venture. The standard deviation of the random-walk proposal was set to 0.5 to roughly match the scale of the problem. To implement the model in Stan, which does not support discrete random variables, we had to manually collapse out (i.e. marginalize over) the is_outlier random variables and inline the marginalized log-density computation into the model program. We used Stan's default NUTS [12] inference engine with default parameters. We also implemented the model using Edward [27]. We were unable to implement the non-vectorized and uncollapsed $\mathrm{MH}+\mathrm{Gibbs}$ algorithm in Edward. Instead, we used Edward's HMC implementation with a manually vectorized and collapsed version of the model (HMC step size 0.01 and 10 steps per proposal). Finally, for a fairer comparison against the manually collapsed algorithms in the other languages, we also implemented a manually collapsed variant of the $\mathrm{MH}+\mathrm{Gibbs}$ algorithm in Gen and Venture, denoted ' $\mathrm{MH}$ '. We also evaluated a hand-coded Julia implementation of the manually collapsed ' $\mathrm{MH}$ ' algorithm.

We ran 20 replicates of each implementation for various numbers of iterations, and computed the median running time, and median predictive log likelihood given the last state in the Markov chain across replicates. Figure 2 shows the resulting time-accuracy profile for each implementation. We also empirically determined the optimum value of predictive log likelihood based on observing convergence of each implementation to the same value, and found the minimum median runtime in milliseconds needed to obtain within two nats of the optimum value. The uncollapsed Venture implementation failed to reach the required predictive likelihood range in the time available. The results are shown in Table 1.
Table 1. Median time (in ms) needed to reach the accuracy threshold, which is two nats below the optimum value. The threshold is shown as a dashed line in Figure 2.

\begin{tabular}{lll} 
System & Algorithm & ms per run \\
\hline hand-coded & MH (manually collapsed) & 26.9 \\
Gen & MH (manually collapsed) & 39.4 \\
Stan & NUTS (manually collapsed) & 79.0 \\
Edward & HMC (manually collapsed) & 87.7 \\
Gen & MH+Gibbs & 125.5 \\
Venture & MH (manually collapsed) & $297,096.2$
\end{tabular}

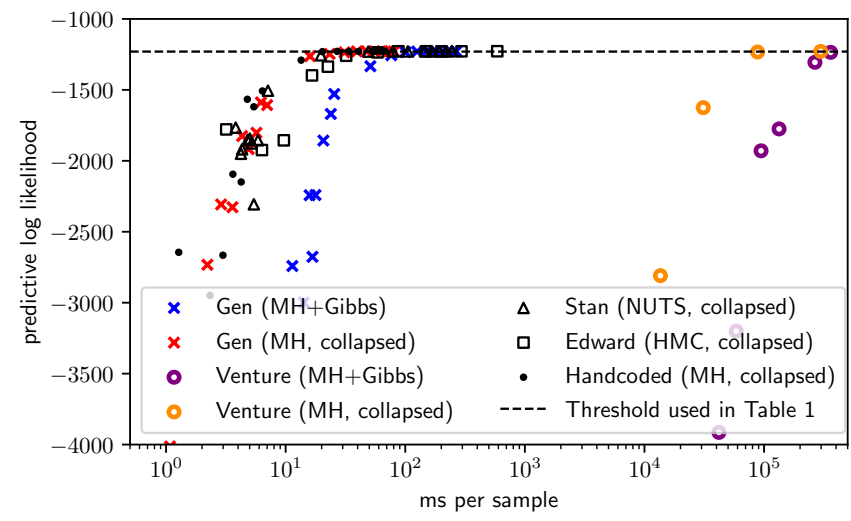

Figure 2. Time/accuracy profiles of inference programs implemented in different probabilistic programming platforms, on a robust Bayesian regression inference problem.

The results show that Gen outperforms Venture by a factor of $10^{3}-10^{4}$, illustrating the benefits of static code-generation and high-performance trace data types. We also found that the performance of Gen is comparable with significantly less expressive systems. Gen and Stan gave comparable performance, despite the fact that Gen is significantly more expressive than Stan, both with regards to custom inference programming, and the modeling language. Whereas Stan uses a built-in inference engine that supports a handful of inference algorithms, Gen supports inference programming, using composable inference tactics. With regards to model expressiveness, Gen can include arbitrary Julia code, whereas Stan is restricted to models for which the unnormalized density can be computed, and models without discrete random variables. Gen also performed similarly to Edward, which statically compiles inference algorithms to TensorFlow computations. Like Stan, Edward is more limited than Gen in terms of model expressiveness-model computations need to be expressed using TensorFlow operations. Note, however, that this evaluation does not exercise Edward's scalable deep learning integration. Finally, the results show that Gen was the only system that gave accurate results within a reasonable time without requiring the discrete variables to be 
manually collapsed out or the model to be vectorized across data points.

\section{Related Work}

Several probabilistic programming projects include compilers for individual inference tactics. Examples include the Swift compiler for BLOG [31]; the built-in Stan compiler [3]; and the Shred compiler for tracing and slicing MCMC updates in Church programs [32]. One important difference between these projects and Gen is that they do not allow the developer to customize the inference strategy based on the problem at hand. A second difference is that Gen models can include arbitrary Julia code that makes stochastic choices.

Recently, the AugurV2 project [13] introduced compiler infrastructure that can be applied to a broader class of Markov chain Monte Carlo algorithms. However, AugurV2 is a standalone modeling language, and does not support models that embed arbitrary Julia code with stochastic choices. Also, in AugurV2, the structure of inference strategy - i.e. the choice of what type of inference tactic to apply to each variable - is synthesized by the system. These choices cannot be customized by the developer to meet the functional requirements of a given application.

Several projects have introduced dynamic approaches to improving the performance of certain classes of inference algorithms. Examples include runtime dependency tracking [15] and callsite caching for continuation-based implementations of Monte Carlo inference [24]. It is technically possible to implement custom inference code generators in Gen that make use of runtime support for these dynamic approaches to improving inference performance. It could be fruitful to explore how Gen's static trace types and dependency analysis could be leveraged to increase the precision or reduce the computational cost of these dynamic approaches.

Like Gen, the Turing probabilistic language [4] is also embedded in Julia. However, Turing does not employ static analysis for inference of specialized trace types or for code generation of specialized inference tactics.

Finally, Gen's inference tactics are inspired by those of Venture [15]. However, unlike Gen, Venture uses runtime dependency tracking, which incurs high overhead. Gen's internal code generation API is based on Venture's low-level primitives, so Gen's architecture allows for the full set of Venture inference tactics to be implemented using code generators that use static analysis to produce fast implementations with low overhead.

\section{Acknowledgments}

This research was supported in part by the US Department of the Air Force contract FA8750-17-C-0239, grants from the MIT Media Lab/Harvard Berkman Center Ethics \& Governance of AI Fund and the MIT CSAIL Systems that Learn Consortium, a gift from the Aphorism Foundation, and the the US Department of Defense through the the National Defense Science \& Engineering Graduate Fellowship (NDSEG) Program.

\section{References}

[1] Martín Abadi, Paul Barham, Jianmin Chen, Zhifeng Chen, Andy Davis, Jeffrey Dean, Matthieu Devin, Sanjay Ghemawat, Geoffrey Irving, Michael Isard, et al. 2016. TensorFlow: A System for Large-Scale Machine Learning.. In OSDI, Vol. 16. 265-283.

[2] Christophe Andrieu, Nando De Freitas, Arnaud Doucet, and Michael I Jordan. 2003. An introduction to MCMC for machine learning. Machine learning 50, 1-2 (2003), 5-43.

[3] Bob Carpenter, Andrew Gelman, Matt Hoffman, Daniel Lee, Ben Goodrich, Michael Betancourt, Michael A. Brubaker, Jiqiang Guo, Peter Li, and Allen Riddell. 2016. Stan: A probabilistic programming language. Journal of Statistical Software 20, 2 (2016), 1-37.

[4] Hong Ge, Kai Xu, and Zoubin Ghahramani. 2018. Turing: Composable inference for probabilistic programming. In International Conference on Artificial Intelligence and Statistics. 1682-1690.

[5] Andrew Gelman, John B Carlin, Hal S Stern, David B Dunson, Aki Vehtari, and Donald B Rubin. 2014. Bayesian data analysis. Vol. 2. CRC press Boca Raton, FL.

[6] Noah D. Goodman, Vikash K. Mansinghka, Daniel M. Roy, Keith Bonawitz, and Joshua B. Tenenbaum. 2008. Church: a language for generative models. In UAI 2008, Proceedings of the 24th Conference in Uncertainty in Artificial Intelligence, Helsinki, Finland, fuly 9-12, 2008.

[7] Noah D. Goodman and Andreas Stuhlmüller. 2014. The design and implementation of probabilistic programming languages. Retrieved 2015/1/16, from http://dippl. org.

[8] Andrew D. Gordon, Thore Graepel, Nicolas Rolland, Claudio Russo, Johannes Borgstrom, and John Guiver. 2014. Tabular: a schema-driven probabilistic programming language. In ACM SIGPLAN Notices, Vol. 49. ACM, 321-334.

[9] Andrew D. Gordon, Thomas A. Henzinger, Aditya V. Nori, and Sriram K. Rajamani. 2014. Probabilistic programming. In Proceedings of the on Future of Software Engineering. ACM, 167-181.

[10] Maria I Gorinova, Andrew D Gordon, and Charles Sutton. 2018. SlicStan: Improving Probabilistic Programming using Information Flow Analysis. Workshop on Probabilistic Programming Semantics (2018).

[11] Thomas L Griffiths, Charles Kemp, and Joshua B Tenenbaum. [n. d.]. Bayesian models of cognition.

[12] Matthew D Hoffman and Andrew Gelman. 2014. The No-U-turn sampler: adaptively setting path lengths in Hamiltonian Monte Carlo. fournal of Machine Learning Research 15, 1 (2014), 1593-1623.

[13] Daniel Huang, Jean-Baptiste Tristan, and Greg Morrisett. 2017. Compiling Markov Chain Monte Carlo Algorithms for Probabilistic Modeling. In Proceedings of the 38th ACM SIGPLAN Conference on Programming Language Design and Implementation (PLDI 2017). ACM, New York, NY, USA, 111-125.

[14] Uber AI Labs. 2017. Pyro, a deep probabilistic programming language. (2017). http://pyro.ai/

[15] Vikash Mansinghka, Daniel Selsam, and Yura Perov. 2014. Venture: a higher-order probabilistic programming platform with programmable inference. arXiv preprint arXiv:1404.0099 (2014).

[16] Brian Milch, Bhaskara Marthi, Stuart Russell, David Sontag, Daniel L. Ong, and Andrey Kolobov. 2007. BLOG: Probabilistic models with unknown objects. Statistical Relational Learning (2007), 373.

[17] Kevin P Murphy, Antonio Torralba, and William T Freeman. 2004. Using the forest to see the trees: A graphical model relating features, objects, and scenes. In Advances in neural information processing systems. 1499-1506.

[18] Siddharth Narayanaswamy, T. Brooks Paige, Jan-Willem van de Meent, Alban Desmaison, Noah Goodman, Pushmeet Kohli, Frank Wood, and 
Philip Torr. 2017. Learning Disentangled Representations with SemiSupervised Deep Generative Models. In Advances in Neural Information Processing Systems. 5927-5937.

[19] Adam Paszke, Sam Gross, Soumith Chintala, Gregory Chanan, Edward Yang, Zachary DeVito, Zeming Lin, Alban Desmaison, Luca Antiga, and Adam Lerer. 2017. Automatic differentiation in PyTorch. (2017).

[20] Judea Pearl. 2014. Probabilistic reasoning in intelligent systems: networks of plausible inference. Elsevier.

[21] Avi Pfeffer. 2001. IBAL: A probabilistic rational programming language. In IfCAI. Citeseer, 733-740.

[22] Avi Pfeffer. 2009. Figaro: An object-oriented probabilistic programming language. Charles River Analytics Technical Report 137 (2009), 96.

[23] Sebastian Robert Riedel, Sameer Singh, Vivek Srikumar, Tim Rocktäschel, Larysa Visengeriyeva, and Jan Noessner. 2014. WOLFE: Strength Reduction and Approximate Programming for Probabilistic Programming.. In AAAI Workshop: Statistical Relational Artificial Intelligence.

[24] Daniel Ritchie, Andreas Stuhlmüller, and Noah Goodman. 2016. C3: Lightweight incrementalized MCMC for probabilistic programs using continuations and callsite caching. In Artificial Intelligence and Statistics. 28-37.

[25] David J. Spiegelhalter, Andrew Thomas, Nicky G. Best, Wally Gilks, and D. Lunn. 1996. BUGS: Bayesian inference using Gibbs sampling.
Version 0.5,(version ii) http://www. mrc-bsu. cam. ac. uk/bugs 19 (1996).

[26] Sebastian Thrun, Wolfram Burgard, and Dieter Fox. 2005. Probabilistic robotics. MIT press.

[27] Dustin Tran, Matthew D. Hoffman, Rif A. Saurous, Eugene Brevdo, Kevin Murphy, and David M. Blei. 2017. Deep Probabilistic Programming. arXiv:1701.03757 [cs, stat] (Jan. 2017). arXiv: 1701.03757.

[28] Jean-Baptiste Tristan, Daniel Huang, Joseph Tassarotti, Adam C Pocock, Stephen Green, and Guy L Steele. 2014. Augur: Data-Parallel Probabilistic Modeling. In Advances in Neural Information Processing Systems 27. 2600-2608.

[29] David Wingate, Andreas Stuhlmüller, and Noah Goodman. 2011. Lightweight implementations of probabilistic programming languages via transformational compilation. In Proceedings of the Fourteenth International Conference on Artificial Intelligence and Statistics. 770-778.

[30] Frank Wood, Jan Willem Meent, and Vikash Mansinghka. 2014. A new approach to probabilistic programming inference. In Artificial Intelligence and Statistics.

[31] Yi Wu, Lei Li, Stuart Russell, and Rastislav Bodik. 2016. Swift: Compiled inference for probabilistic programming languages. arXiv preprint arXiv:1606.09242 (2016).

[32] Lingfeng Yang, Patrick Hanrahan, and Noah Goodman. 2014. Generating efficient MCMC kernels from probabilistic programs. In Artificial Intelligence and Statistics. 1068-1076. 